1994-08-01

\title{
Performance analysis of antennas for hand-held transceivers using FDTD
}

Michael A. Jensen

jensen@byu.edu

Yahya Rahmat-Samii

Follow this and additional works at: https://scholarsarchive.byu.edu/facpub

Part of the Electrical and Computer Engineering Commons

\section{Original Publication Citation}

Jensen, M. A., and Y. Rahmat-Samii. "Performance Analysis of Antennas for Hand-Held

Transceivers using FDTD." Antennas and Propagation, IEEE Transactions on 42.8 (1994): 116-13

\section{BYU ScholarsArchive Citation}

Jensen, Michael A. and Rahmat-Samii, Yahya, "Performance analysis of antennas for hand-held transceivers using FDTD" (1994). Faculty Publications. 698.

https://scholarsarchive.byu.edu/facpub/698

This Peer-Reviewed Article is brought to you for free and open access by BYU ScholarsArchive. It has been accepted for inclusion in Faculty Publications by an authorized administrator of BYU ScholarsArchive. For more information, please contact ellen_amatangelo@byu.edu. 


\title{
Performance Analysis of Antennas for Hand-Held Transceivers Using FDTD
}

\author{
Michael A. Jensen, Student Member, IEEE, and Yahya Rahmat-Samii, Fellow, IEEE
}

\begin{abstract}
The design of antennas for hand-held communications devices depends on the implementation of simulation tools that can accurately model general topologies. This work presents the analysis of small antennas mounted on hand-held transceivers using the finite-difference time-domain (FDTD) method. The key features of the FDTD implementation are discussed, with particular emphasis placed upon modeling of the source region. The technique is used to predict the gain patterns and broadband input impedance behavior of monopole, planar inverted $F$, and loop antenna elements mounted on the handset. Effects of the conducting handset chassis, the plastic casing around the device, and lumped elements integrated into the antenna design are illustrated. Experimental results are provided to verify the accuracy of the computational methodology. The concept of antenna diversity is discussed, and key assumptions and expressions are provided that characterize the multipath fading fields. Several computational examples demonstrate the diversity performance of two receiving antennas on a single handset.
\end{abstract}

\section{INTRODUCTION}

$\mathbf{T}$ HE recent efforts aimed at improving available personal communications services have generated an increased interest in the performance of compact antenna structures mounted on hand-held devices. The characterization of such antennas is dependent upon the development of simulation tools that can accurately model general topologies, including wires, dielectrics, conductors, and lumped elements. In an effort to meet these simulation needs, attention has been focused on the use of the finite-difference time-domain (FDTD) [1], [2] methodology for antenna analysis [3]-[14]. Although past contributions in this arena have demonstrated the effectiveness of the FDTD approach in characterizing antenna configurations, only a limited amount of research has appeared relating to the simulation of practical antenna geometries operating in their true radiating environment.

This paper presents an investigation of the monopole, the planar inverted $F$ antenna (PIFA) [15], and the loop antenna [16] (see Fig. 1) mounted on a hand-held transceiver using the FDTD technique. The radiation pattern, gain, and input impedance for these radiators are computed for several different topologies to illustrate the effects of the handset conducting chassis, the plastic casing surrounding the unit, and passive lumped elements integrated into the design on the antenna performance. A new source model is proposed that considerably reduces the time required to obtain the transient

Manuscript received June 1, 1993; revised February 17, 1994. This work was supported by ARPA under Contract DAAB07-93-C-C501.

The authors are with the Electrical Engineering Department, University of California, Los Angeles, CA, 90024-1594 USA

IEEE Log Number 9403658

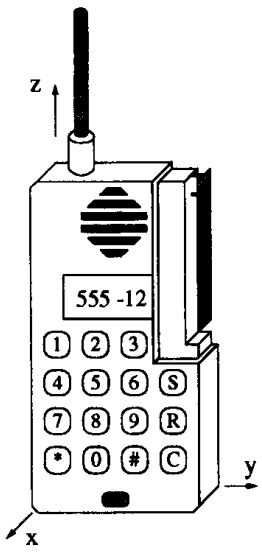

(a)

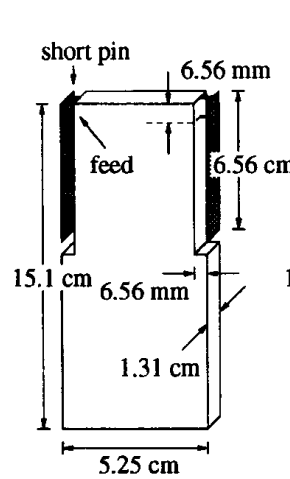

(c)

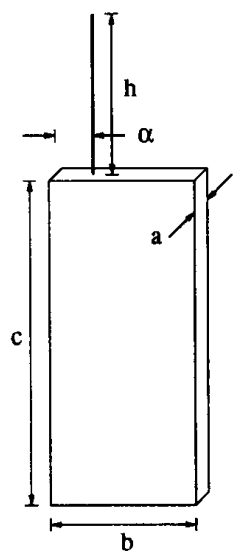

(b)

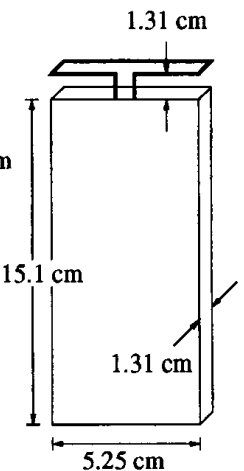

(d)
Fig. 1. (a) Typical handset geometry showing the monopole and PIFA elements. Configuration and dimensions for the (b) monopole, (c) dual PIFA, and (d) top-mounted loop on the handset.

antenna response. Experimental data is provided for several of the configurations to validate the effectiveness of the FDTD for this class of problems. Due to page limitations, issues such as the interaction with the operator's tissue can not be detailed in this paper and as such are left to other publications [17].

Because hand-held communications equipment must often operate in a multipath fading environment, efforts to improve the data transfer reliability have motivated the implementation of antenna diversity schemes in these devices [18]-[24]. Therefore, the concept of antenna diversity is discussed, and key equations and assumptions used to describe the multipath 
phenomenon are provided. Computational results are presented that illustrate the diversity performance of multiple small antennas mounted on a single handset.

\section{FDTD IMPLEMENTATION}

The FDTD [1] technique is a numerical approach that uses discrete approximations of Maxwell's time domain equations. The resulting algebraic equations can be used to track the time evolution of the fields within a given spatial region. The derivation as well as the practical implementation of this algorithm are well covered in past literature [1]-[14] and as such will not be covered in this paper.

In this work, the FDTD algorithm has been implemented to allow modeling and simulation of 1) very general radiating element geometries with dielectrics, wires, and planar conductors, 2) the conducting handset chassis, 3) the plastic casing surrounding the device, 4) the antenna feed, 5) lumped elements for tuning, and 6) multiple radiators for diversity. The technique is applied with Mur's second-order absorbing boundary condition at the outer computational domain boundary [11]. Far-zone gain and radiation patterns are computed using an integral near- to far-field transformation equation [12], [13]. The FDTD notation used in the following is similar to that provided in [9]. Note that $\Delta x, \Delta y$, and $\Delta z$ denote the spatial grid discretization sizes and $\Delta t$ represents the time step size.

\section{A. Antenna Source Implementation}

Typically, the excitation for wire-fed antenna geometries within the FDTD framework is performed using a gap voltage model in which a voltage is introduced in one cell of the feeding wire [3]. For a $z$-directed wire, the excitation electric field relates to the source voltage $V_{s}(t)$ through the expression

$$
V_{s}(n \Delta t)=-E_{z, i, j, k}^{n} \Delta z
$$

where the cell at $(i, j, k)$ is located at the antenna feed point.

In this work, we present a new feed model that simulates a coaxial feeding cable. As seen in the side view of the coax illustrated in Fig. 2, a gap voltage is introduced in the coaxial center conductor, and the standard FDTD relations are used within the coax to propagate the fields toward the antenna. At the end of the coax, special interfacing relations are required to update the radial electric and circumferential magnetic fields. The relation for the circumferential magnetic field $H_{x, i, j, k}$ shown in Fig. 2 is derived using the integral forms of Maxwell's equations and assuming a $1 / \rho$ dependence of the fields, as discussed in [8]. Using this approach, the modified time-stepping equation assumes the form

$$
\begin{aligned}
H_{x, i, j, k}^{n+1 / 2}= & H_{x, i, j, k}^{n-1 / 2} \\
& +\frac{\Delta t}{\mu \Delta z}\left[E_{y, i, j, k}^{n}-E_{y, i, j, k-1}^{n} \frac{r_{b}}{\Delta y} \frac{\ln \left(r_{b} / r_{a}\right)}{\ln \left(\Delta y / r_{a}\right)}\right] \\
& -\frac{\Delta t}{\mu(\Delta y / 2) \ln \left(\Delta y / r_{a}\right)}\left[E_{z, i, j, k}^{n}-E_{z, i, j-1, k}^{n}\right]
\end{aligned}
$$

where $r_{a}$ and $r_{b}$ represent the radii of the coax inner and outer conductors, respectively. When computing the radial electric

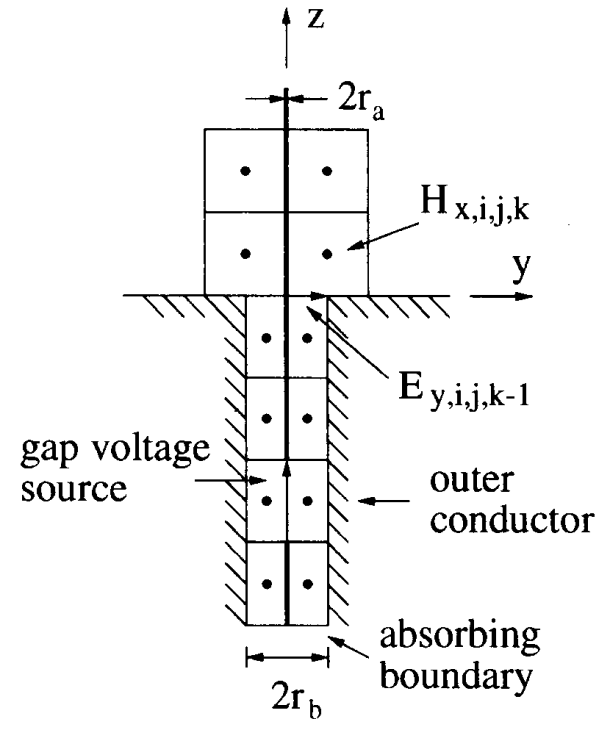

Fig. 2. Cross sectional view of the simulated coax and the grid used to determine the interface time-stepping equations.

field $E_{y, i, j, k-1}$ in Fig. 2, the standard FDTD relations are used with the modification that $H_{x, i, j, k} \rightarrow\left(\Delta y / r_{b}\right) H_{x, i, j, k}$ to align $H_{x, i, j, k}$ with $H_{x, i, j, k-1}$. The parameters $r_{a}, r_{b}$, and $\epsilon$ within the coax are chosen to represent a desired characteristic impedance. Similar relations can be derived for the other circumferential magnetic and radial electric fields at the interface. Because reverse propagating modes in the coax are excited by the source voltage and by reflections from the coax-antenna transition, it is essential to terminate the coax with an absorbing boundary condition, as implied in Fig. 2 .

For the simulated coax, the feed point voltage is obtained from the computed radial electric field using

$$
V_{s}(n \Delta t)=\frac{r_{b}}{2} \ln \left(\frac{r_{b}}{r_{a}}\right) E_{y, i, j, k-1}^{n} .
$$

The current in the wire for both source models can be obtained using a discretized form of Ampere's Law that assumes the form

$$
\begin{aligned}
I_{s}((n+1 / 2) \Delta t)= & \left(H_{x, i, j, k}^{n+1 / 2}-H_{x, i, j+1, k}^{n+1 / 2}\right) \Delta x \\
& +\left(H_{y, i+1, j, k}^{n+1 / 2}-H_{y, i, j, k}^{n+1 / 2}\right) \Delta y .
\end{aligned}
$$

The time offset of $\Delta t / 2$ between the source voltage and current is generally neglected since it is assumed to be small.

Both source models discussed above appear to give virtually identical results in terms of input impedance and antenna gain. However, the simulated coaxial model provides two distinct advantages. First, it allows examination of the antenna input impedance at a point within the feeding transmission line rather than solely at the coax-antenna transition. Second, it has been observed in this study that the fields near the antenna terminals decay more rapidly in time for the coaxial model 


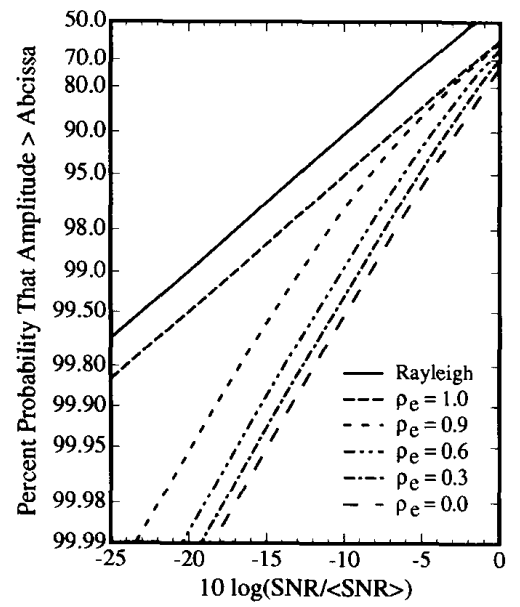

Fig. 3. Cumulative probability distribution for two-branch antenna diversity with maximal ratio combining for several values of the envelope correlation coefficient $\rho_{\epsilon}$. The Rayleigh reference for one antenna is provided for comparison.

than for the gap voltage model. In general, the time required for the terminal current to reach $1 \%$ of its peak value for the simulated coax is $30 \%$ to $60 \%$ of the time required for the standard gap model.

\section{ANTENNA DIVERSITY}

The use of antenna diversity to mitigate the detrimental effects of signal fades in a multipath environment is a topic of considerable interest to designers in the personal wireless communications industry. For two antennas on a single receiver, the diversity performance is most commonly evaluated by investigating the correlation coefficient $\rho_{s}-\mathrm{a}$ statistical value indicating the similarity in the voltages received by the antennas. Because a great deal of literature exists that contains a statistical description of multipath fading fields as well as relations for the correlation coefficient [18]-[24], only a brief presentation relating to these topics will be presented here. In all expressions, the variables $\theta$ and $\phi$ are taken with respect to a coordinate system oriented with the $z$-axis perpendicular to the earth. The following basic assumptions concerning the incoming multipath fields are used in deriving expressions for the value of $\rho_{s}$ :

1) The fading signal envelope is Rayleigh distributed.

2) Orthogonal polarizations are uncorrelated.

3) Each individual polarization is spatially uncorrelated.

4) The field arrives in the horizontal $(\theta=\pi / 2)$ plane only.

5) The time-averaged power density per steradian $\vec{S}(\theta, \phi)=S_{\theta}(\theta, \phi) \hat{\theta}+S_{\phi}(\theta, \phi) \hat{\phi}$ is constant in the horizontal plane such that $\vec{S}(\pi / 2, \phi)=S_{\theta}^{o} \hat{\theta}+S_{\phi}^{o} \hat{\phi}=$ constant.

Using these assumptions, the correlation coefficient for the signals received by the two antennas can be expressed as [18]

$$
\rho_{s}=\frac{\int_{0}^{2 \pi} A_{12}(\phi) d \phi}{\left\{\int_{0}^{2 \pi} A_{11}(\phi) d \phi \int_{0}^{2 \pi} A_{22}(\phi) d \phi\right\}^{1 / 2}}
$$

where

$$
\begin{aligned}
A_{p q}(\phi)= & \Gamma E_{\theta p}(\pi / 2, \phi) E_{\theta q}^{*}(\pi / 2, \phi) \\
& +E_{\phi p}(\pi / 2, \phi) E_{\phi q}^{*}(\pi / 2, \phi)
\end{aligned}
$$

and $\vec{E}_{p}(\theta, \phi)=E_{\theta p}(\theta, \phi) \hat{\theta}+E_{\phi p}(\theta, \phi) \hat{\phi}$ is the vector radiation pattern associated with antenna \#p. The envelope correlation coefficient, which is the measured quantity in antenna diversity experimentation, is approximately the magnitude squared of the voltage coefficient [18], or

$$
\rho_{e} \approx\left|\rho_{s}\right|^{2} \text {. }
$$

The parameter $\Gamma$ in (6) is the cross-polarization discrimination (XPD) [21] of the incident multipath field and is given by

$$
\Gamma=\frac{S_{\theta}^{o}}{S_{\phi}^{o}}
$$

where $S_{\theta}^{o}$ and $S_{\phi}^{o}$ are identified in Assumption 5 above. In an urban environment, it is often assumed that either polarization is equally likely, resulting in a XPD of $\Gamma=0 \mathrm{~dB}$ [18]. However, there is still some speculation concerning the polarization characteristics of the incident field at the mobile antenna [22]-[24]. In light of this, the computations performed for this paper will be presented for a broad range of XPD values.

Fig. 3 shows the cumulative probability distribution of the signal-to-noise ratio (SNR) normalized to its time average $(\langle\mathrm{SNR}\rangle)$ for various values of $\rho_{e}$ and for two branch antenna diversity. Maximal ratio combining, in which the two signals are received, co-phased, properly weighted, and added, is assumed in this plot. Also shown is the Rayleigh distribution that corresponds to a single antenna in the multipath environment. As can be seen, reducing the envelope correlation coefficient improves the probability distribution of the received SNR. For example, when no diversity is used, the Rayleigh distribution predicts that the received normalized SNR will be above -23 $\mathrm{dB} 99.5 \%$ of the time. If two antennas are used with $\rho_{e}=0.6$, the SNR increases to $-12 \mathrm{~dB}$ at the $99.5 \%$ reliability level. In light of this, the goal in diversity antenna design is to minimize $\rho_{e}$ to the extent possible using a combination of spatial, angle, and polarization diversity.

The method for computing the vector patterns $\vec{E}_{1}$ and $\vec{E}_{2}$ depends upon the type of diversity combining used on the received signals. For example, if switched or selection diversity is to be implemented, the pattern $\vec{E}_{1}$ should be determined as the pattern for element $\# 1$ radiating in the presence of element \#2 which is open circuited. For other combining techniques, the pattern should be computed for element \#2 terminated with a matched load (absorbing boundary in the FDTD computation). For these computations, each pattern is obtained for the respective element radiating in the presence of a parasitic rather than excited second antenna in order to model the case of one antenna receiving while the second is positioned in a multipath fade [18]. 


\section{COMPUTATIONAL AND EXPERIMENTAL RESUlTS}

In the following computations, the source function used is a sinusoid modulated by a Gaussian envelope that is expressed as

$$
E(t)=\cos \left(\omega_{o} t\right) e^{-t^{2} / 2 \tau^{2}}
$$

where the parameter $\tau$ controls the pulse width. Because this forcing function results in a frequency spectrum described by a Gaussian centered at $\omega_{o}$ and with variance $1 / \tau^{2}$, it is possible to simply control the center frequency and bandwidth of the excitation. In the computations that follow, $\tau$ is chosen such that $1 / \tau^{2}=$ the bandwidth of interest. For single frequency computations, $\tau \rightarrow \infty$ is used.

The experimental measurements provided have been performed at the University of California, Los Angeles, antenna measurement laboratory. Impedance measurements are obtained from a Hewlett-Packard 8510B network analyzer. Pattern measurements are performed in a small anechoic chamber configured for far-field measurements. In all of the examples, the wire used has a radius $r_{a}$ given by

$$
2 r_{a}=0.9195 \mathrm{~mm}
$$

which corresponds to the radius of the inner conductor for RG402/U $50 \Omega$ semirigid coaxial cable.

\section{A. Monopole Antenna}

The monopole is perhaps the most commonly used antenna for hand-held devices because of its broad-band characteristics and simple construction. Fig. 1(b) illustrates the geometry for such a configuration, where the monopole is centered on the handset in the $x$-direction and is offset a distance $\alpha$ in the $y$-direction. Fig. 4 compares the computed input impedance versus frequency with experimental measurements for the dimensions given in the figure caption. The small handset dimensions result from a frequency scaling that boosts the operating frequency of the device to $6 \mathrm{GHz}$. The computations performed use the simulated coaxial line discussed in Section II. As can be seen, very good agreement exists between the two sets of data, with only slight discrepancies occurring in the reactance near the upper end of the band. These curves also illustrate the broad-band nature of the monopole near its first resonance frequency, where the impedance is nearly matched to a $50 \Omega$ feeding coax.

The effect of the conducting handset chassis on the monopole radiation pattern is an important consideration in the design of antennas for practical applications. Fig. 5 provides the $\hat{\theta}$-polarized patterns normalized to the antenna gain for the monopole on the handset at a frequency of $6 \mathrm{GHz}$. The experimental data shown extends only across the upper hemisphere because brackets in the measurement facility interfered with the lower hemisphere measurements. Here again, good correlation exists between the computed and measured results. For comparison purposes, the gain pattern for a $2.5-\mathrm{cm}$ dipole antenna, whose pattern shape corresponds to that of the monopole on an infinite ground plane, is also shown. As can be seen, the presence of the chassis alters the radiation pattern. Simulations of this nature

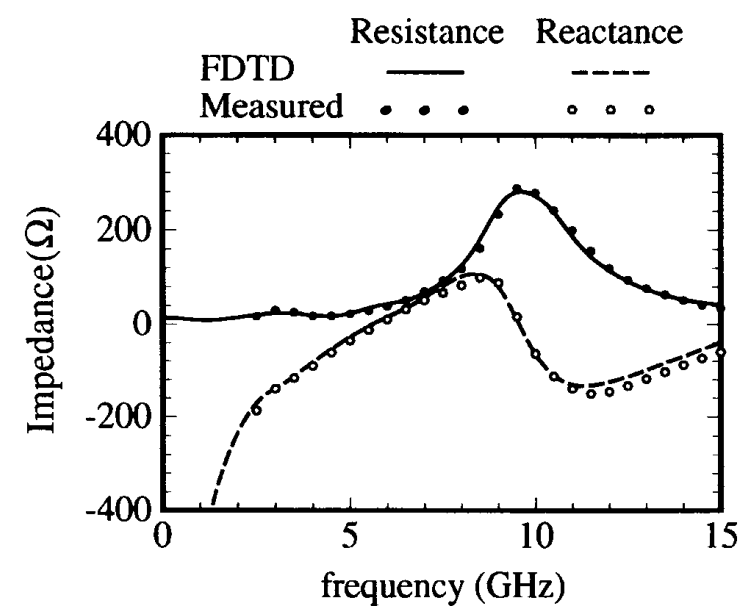

Fig. 4. Computed and measured input impedance versus frequency for the monopole on the handset illustrated in Fig. I(b) for the parameters $a=1 \mathrm{~cm}$ $b=2 \mathrm{~cm}, c=3 \mathrm{~cm}, h=1.25 \mathrm{~cm}$, and $\alpha=1 \mathrm{~cm}$. These small dimensions result from frequency scaling the handset to operate at $6 \mathrm{GHz}$.

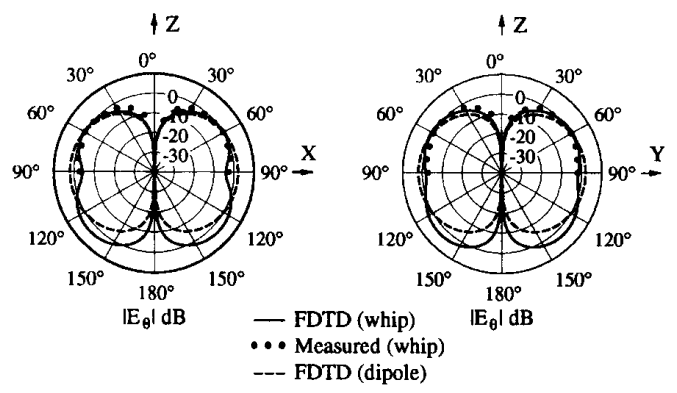

Fig. 5. Computed gain patterns at $6 \mathrm{GHz}$ compared to measured data in the upper hemisphere for the geometry of Fig. 1(b) with the dimensions given in the caption of Fig. 4. The gain patterns for a $2.5-\mathrm{cm}$ dipole are provided for comparison.

provide considerable insight into the radiation behavior of the antenna/transceiver system.

In many applications, an inductive load is integrated into the monopole to improve the match or reduce the antenna size. Fig. 6 illustrates this effect by presenting the variation of $\left|S_{11}\right|$ (assuming a $50 \Omega$ feeding transmission line) with frequency for the monopole of Fig. 1(b). The handset dimensions are again provided in the figure caption. The different curves represent the results when the monopole is alone on the handset, when a plastic casing is present, and when the plastic and a 10$\mathrm{nH}$ inductor one FDTD cell above the monopole feed point are included. The plastic layer is modeled as a $3.28 \mathrm{~mm}$ thick lossless dielectric with permittivity $\epsilon_{r}=2$, which is immediately adjacent to the conducting chassis. An expanded view of the plot about the $900-\mathrm{MHz}$ resonance is provided for clarity. These results show that the plastic exercises relatively little effect on the monopole antenna performance at the lower end of the frequency band and a slightly more significant effect at higher frequencies. Also, the inductive load reduces the resonant frequencies and improves the match, especially at 


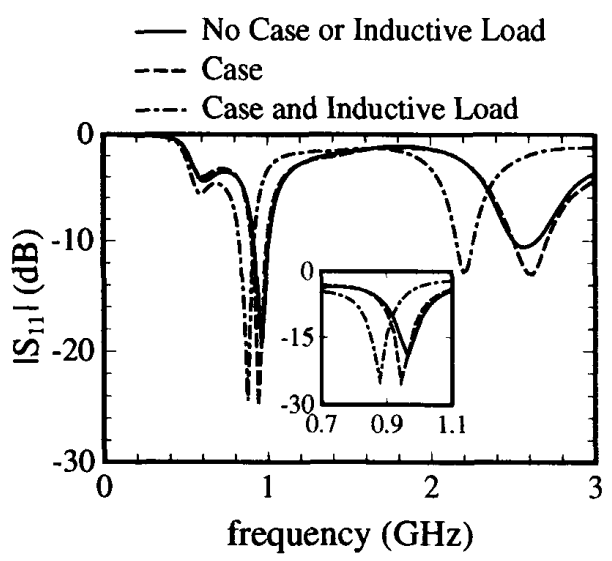

Fig. 6. Effect of the 3.28-mm plastic case and 10-nH inductor on the variation of $\left|S_{11}\right|$ versus frequency for the monopole of Fig. 1(b). The dimensions are $a=1.31 \mathrm{~cm}, b=5.25 \mathrm{~cm}, c=15.1 \mathrm{~cm}, h=8.5 \mathrm{~cm}$, and $\alpha=6.56 \mathrm{~mm}$

the higher frequencies. This capability of modeling lumped elements within the FDTD simulation tool allows the design engineer to efficiently determine proper loading strategies to improve the performance of antennas for communications devices.

\section{B. Planar Inverted F Antenna}

An interesting antenna topology that has received some attention in the literature is the planar inverted $F$ antenna (PIFA), a modified microstrip that allows a simple impedance match in a low-profile design [15], [25], [26]. This geometry is illustrated in Fig. 1(c), where the antenna is mounted on the side of a conducting handset chassis. The short circuit introduced at one edge of the radiating element allows the antenna size to be reduced by a factor of two for a given resonant frequency. Past observations have revealed that the PIFA offers bandwidths as high as $10 \%$ when it is located on a small ground plane or conducting chassis [15].

Fig. 7(a) depicts the geometry for a PIFA element on a finite ground plane. The input impedance variation with frequency for this configuration is illustrated in Fig. 7(b). Measured data obtained from a prototype of this antenna configuration is also presented in the figure. As can be observed, very good agreement exists between the two sets of data. This plot also demonstrates the excellent match between the antenna and coax near $900 \mathrm{MHz}$.

For an integrated antenna such as the PIFA, the effect of the plastic case that encloses the handset is an important consideration. Fig. 8 illustrates this by presenting the input impedance versus frequency for the PIFA of Fig. 1(c) with and without the plastic casing. Referring to Fig. 1(c), the antenna located toward the positive $y$-axis is excited while the second is terminated with an absorbing boundary. The plastic layer is again a $3.28 \mathrm{~mm}$ thick lossless dielectric with permittivity $\epsilon_{r}=2$. These results reveal that the dielectric loading by the casing noticeably reduces the antenna resonant frequency and therefore allows the element size to be reduced for a given

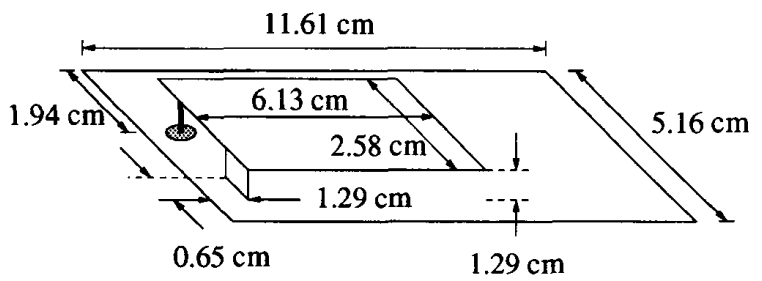

(a)

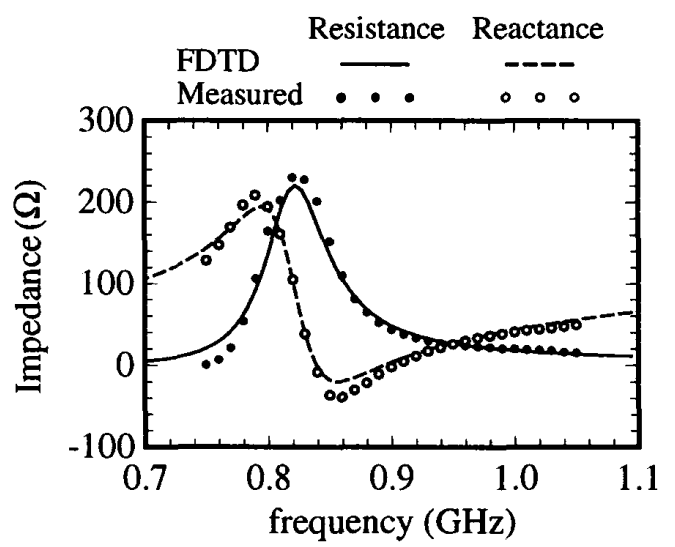

(b)

Fig. 7. PIFA on a finite ground plane: (a) geometry and (b) computed and measured input impedance versus frequency.

operating frequency. Fig. 9 represents the gain patterns at 915 $\mathrm{MHz}$ in the principal planes for the PIFA element of Fig. 1(c) with the plastic casing. As can be seen, the PIFA provides a pattern very similar to that of the monopole.

\section{Loop Antenna}

The loop antenna is an element that has often been used in pager devices, but to date it has found very little application in hand-held transceivers. This is probably because the small loop is highly inductive and difficult to match to the feed line. However, as the operating frequency of wireless communications devices moves into higher bands, the loop becomes a viable antenna element for these applications, particularly in designs where balanced amplifiers must interface with the antenna. Fig. 1(d) illustrates the geometry for a wire loop mounted on the top of a handset. Fig. 10 presents the input impedance variation for this configuration both with and without the $3.28 \mathrm{~mm}$ thick plastic casing, which completely encloses the handset and antenna. Once again, the dielectric lowers the resonance frequencies of the radiator. This plot further shows that though the loop presents challenging matching requirements at low frequencies, its impedance characteristics are well behaved near $2 \mathrm{GHz}$, where the loop perimeter approaches $1 \lambda$.

\section{Diversity Performance of Dual Antennas}

Determining the diversity performance of two antennas mounted on a handset requires computation of the envelope 


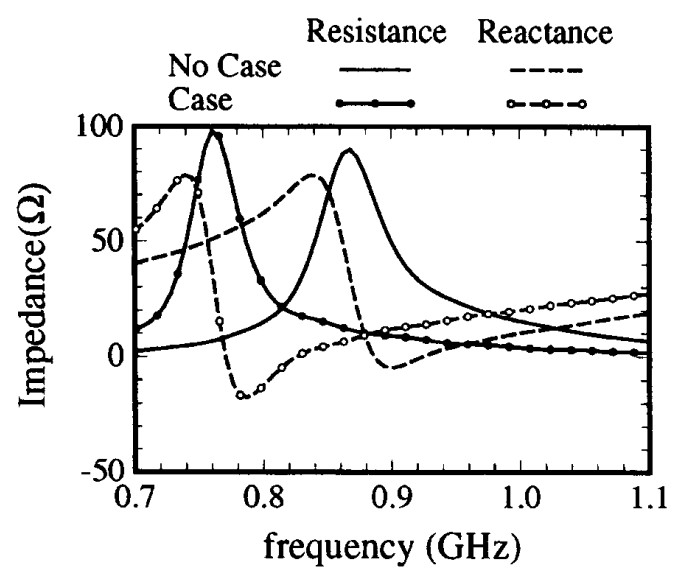

Fig. 8. Input impedance versus frequency for the side-mounted PIFA in Fig. 1 (c) with and without a 3.28 -mm plastic casing enclosing the handset.

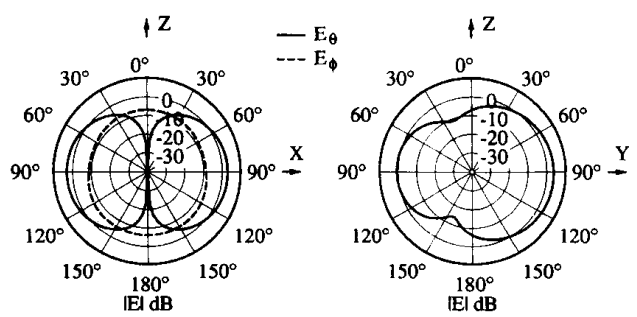

Fig. 9. Gain patterns at $915 \mathrm{MHz}$ for the side-mounted PIFA of Fig. 1(c) with the $3.28-\mathrm{mm}$ plastic casing.

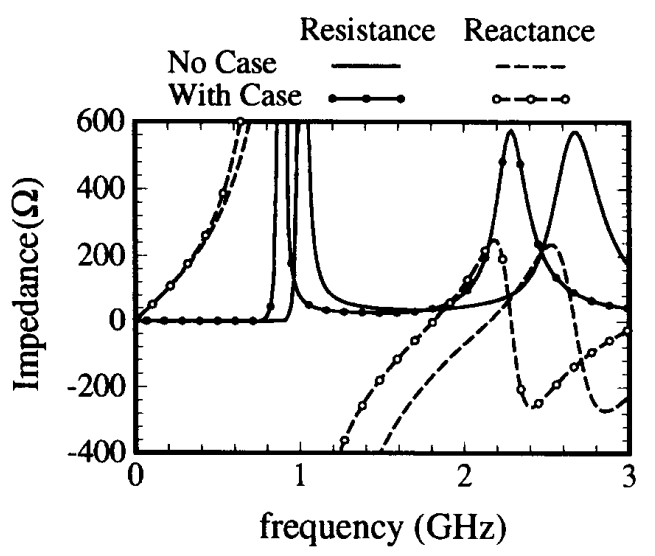

Fig. 10. Input impedance versus frequency for the loop on the handset illustrated in Fig. 1(d) with and without the $3.28-\mathrm{mm}$ plastic casing.

correlation coefficient. Consistent with assumption 4 in Section III, it is assumed that the incident multipath field is confined to a plane parallel with the horizon. To illustrate the effects of varied orientation during operation, the handset coordinate system is allowed to rotate about its $x$-axis such that its $z$-axis makes an angle $\beta$ with the perpendicular to the horizon. As a first example of this computation, the dual PIFA geometry of

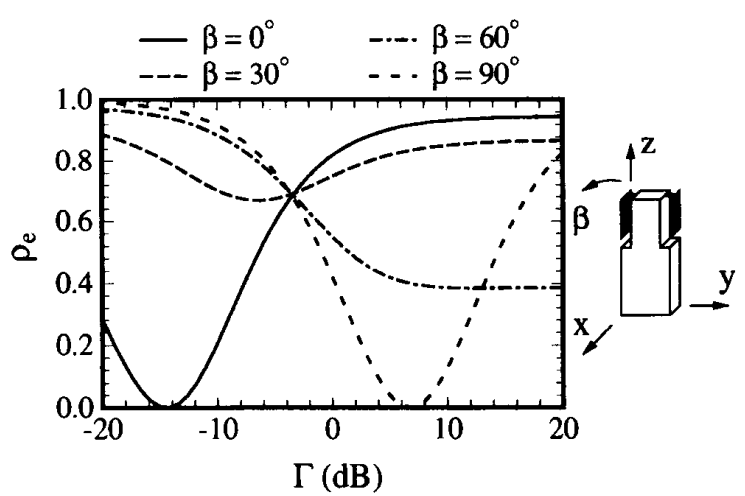

Fig. 11. Envelope correlation coefficient versus the XPD $(\Gamma)$ at $915 \mathrm{MHz}$ for the dual PIFA configuration shown in Fig. 1(c) for several different handset orientations.

Fig. 1(c) is used with the plastic casing included. The radiation patterns at $915 \mathrm{MHz}$ computed for each antenna (with the other antenna terminated in a matched load) are incorporated into (5) and (7) to obtain $\rho_{e}$. The resulting values of $\rho_{e}$ are illustrated versus the XPD $(\Gamma)$ of the incident multipath field in Fig. 11 for several values of $\beta$. The dependence of $\rho_{e}$ on $\Gamma$ implies that significant decorrelation is produced using polarization diversity between the two branches. Also noteworthy is the influence of pattern diversity as the handset is rotated.

A second interesting example is the combination of the monopole in Fig. 1(b) with one of the PIFA elements in Fig. 1(c), as implied by Fig. 1(a). The monopole dimensions are those used in the caption of Fig. 6, and the plastic casing is again included. The results are shown in Fig. 12 at $915 \mathrm{MHz}$ versus $\Gamma$ for several different handset orientations. Note that in this case the value of $\rho_{e}$ is lower for $\Gamma=0 \mathrm{~dB}$ than for the case of the dual PIFA antenna studied in Fig. 11. This improvement is attributed to the fact that, in contrast to the dual PIFA configuration, the two elements used for Fig. 12 exhibit different pattern and polarization characteristics.

As a demonstration of the use of Figs. 11 and 12 for determining the benefits of the diversity schemes, consider the curve for $\beta=30^{\circ}$ in Fig. 12, which predicts that $\rho_{e} \approx 0.3$ at $\Gamma=0 \mathrm{~dB}$. Using this value in Fig. 3 suggests that the received SNR for the diversity configuration is approximately $12 \mathrm{~dB}$ higher than that for a single antenna in a Rayleigh fading environment. Studies such as this aid the antenna designer in selecting antenna configurations that will be effective in combating rapid fading due to multipath delays in urban environments.

\section{CONCLUSION}

In this work we have used the FDTD method to accurately characterize the performance of single- and dual-antenna configurations mounted on hand-held communications devices. The key features of the FDTD implementation have been presented, with special consideration given to the source models used. The concept of antenna diversity has also been 


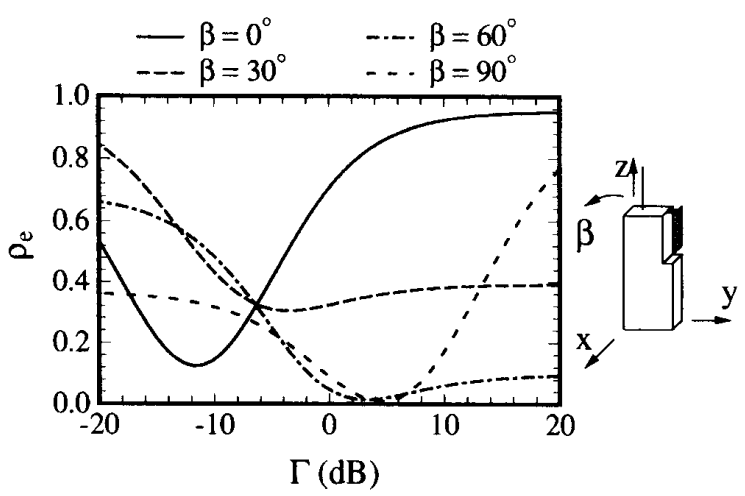

Fig. 12. Envelope correlation coefficient versus the XPD $(\Gamma)$ at $915 \mathrm{MHz}$ for the PIFA-monopole configuration shown in Fig. 1(a) for several different handset orientations.

discussed, and an expression relating the envelope correlation coefficient to the radiation patterns of two coupled antennas has been provided. The geometrical flexibility of the FDTD algorithm has allowed simulation of the monopole, PIFA, and loop elements operating in the presence of a conducting chassis and plastic casing similar to what might form the body of a transceiver handset. Comparisons have demonstrated that the FDTD results match very well with measured data for both radiation patterns and broad-band input impedance. We have also demonstrated the use of the FDTD tool in determining the diversity performance of two antennas operating simultaneously on the handset.

\section{ACKNOWLEDGMENT}

The authors thank J. Colburn for his help in obtaining the experimental data.

\section{REFERENCES}

[1] K. S. Yee, "Numerical solution of initial boundary value problems involving Maxwell's equations in isotropic media," IEEE Trans. Antennas Propagat., vol. AP-14, pp. 302-307, May 1966.

[2] A. Taflove and M. E. Brodwin, "Numerical solution of steady-state electromagnetic scattering problems using the time-dependent Maxwell's equations," IEEE Trans. Microwave Theory Tech., vol. MTT-23, pp. 623-630, Aug. 1975.

[3] R. Luebbers, L. Chen, T. Uno, and S. Adachi, "FDTD calculation of radiation patterns, impedance, and gain for a monopole antenna on a conducting box," IEEE Trans. Antennas Propagat., vol. 40, pp. 1577-1583, Dec. 1992.

[4] J. G. Maloney, G. S. Smith, and W. R. Scott, Jr., "Accurate computation of the radiation from simple antennas using the finite-difference time-domain method," IEEE Trans. Antennas Propagat., vol, 38, pp. 1059-1068, July 1990.

[5] A. Reineix and B. Jecko, "Analysis of microstrip patch antennas using finite difference time domain method," IEEE Trans. Antennas Propagat., vol. 37 , pp. 1361-1369, Nov. 1989

[6] D. S. Katz, M. J. Piket-May, A. Taflove, and K. R. Umashankar, "FDTD analysis of electromagnetic wave radiation from systems containing horn antennas," IEEE Trans. Antennas Propagat., vol. 39, pp. 1203-1212, Aug. 1991.

[7] P. A. Tirkas and C. A. Balanis, "Finite-difference time-domain method for antenna radiation," IEEE Trans. Antennas Propagat., vol. 40, pp. 334-340, Mar. 1992.

[8] A. Taflove, K. R. Umashankar, B. Beker, F. Harfoush, and K. S. Yee, "Detailed FD-TD analysis of electromagnetic fields penetrating narrow slots and lapped joints in thick conducting screens," IEEE Trans. Antennas Propagat, vol. 36, pp. 247-257, Feb. 1988

[9] D. M. Sheen, S. M. Ali, M. D. Abouzahra, and J. A. Kong, "Application of the three-dimensional finite-difference time-domain method to the analysis of planar microstrip circuits," IEEE Trans. Microwave Theory Tech., vol. 38, pp. 849-857, July 1990.

[10] W. Sui, D. A. Christensen, and C. H. Durney, "Extending the twodimensional FDTD method to hybrid electromagnetic systems with active and passive lumped elements," IEEE Trans. Microwave Theory Tech., vol. 40, pp. 724-730, Apr. 1992.

[11] G. Mur, "Absorbing boundary conditions for the finite-difference approximation of the time-domain electromagnetic field equations," IEEE Trans. Electromagn. Compat., vol. EMC-23, pp. 377-382, Nov. 1981.

[12] R. J. Luebbers, K. S. Kunz, M. Schneider, and F. Hunsberger, "A finitedifference time-domain near zone to far zone transformation," IEEE Trans. Antennas Propagat., vol. 39, pp. 429-433, Apr. 1991.

[13] R. J. Luebbers and J. Beggs, "FDTD calculation of wide-band antenna gain and efficiency," IEEE Trans. Antennas Propagat., vol. 40, pp. 1403-1407, Nov. 1992

[14] M. A. Jensen and Y. Rahmat-Samii, "FDTD analysis of PIFA diversity antennas on a hand-held transceiver unit," in 1993 IEEE AP-S Int. Symp. Dig., Ann Arbor, MI, June 27-July 2, 1993, vol. 2, pp. 814-817.

[15] T. Taga, "Analysis of planar inverted $F$ antennas and antenna design for portable radio equipment," in Analysis, Design, and Measurement of Small and Low Profile Antennas, K. Hirasawa and M. Haneishi, Eds. Boston: Artech, 1992, ch. 5.

[16] M. A. Jensen and Y. Rahmat-Samii, "Characterisation of electromagnetically coupled superquadric loop antennas for mobile communications applications," IEE Proc. H, vol. 141, pp. 85-93, Apr. 1994.

[17] "The electromagnetic interaction between biological tissue and antennas on a transceiver handset," in 1994 IEEE AP-S Int. Symp. Dig. Seattle, WA, June 19-24, 1994, pp. 367-370.

[18] R. G. Vaughan and J. B. Andersen, "Antenna diversity in mobile communications," IEEE Trans. Veh. Technol., vol. VT-36, pp. 149-172, Nov. 1987.

[19] W. C. Jakes, Jr., Microwave Mobile Communications. New York: Wiley, 1974.

[20] R. G. Vaughan, "Polarization diversity in mobile communications," IEEE Trans. Veh. Technol., vol. 39, pp. 177-186, Aug. 1990

[21] P. C. F. Eggers, J. Toftgard and A. M. Oprea, "Antenna systems for base station diversity in urban small and micro cells," IEEE J. Select. Areas Commun., vol. 11, pp. 1046-1057, Sept. 1993.

[22] S. Kozono, T. Tsuruhara, and M. Sakamoto, "Base station polarization diversity reception for mobile radio," IEEE Trans. Veh. Technol., vol. VT-33, pp. 301-306, Nov. 1984.

[23] W. C. Y. Lee and Y. S. Yeh, "Polarization diversity system for mobile radio," IEEE Trans. Commun., vol. COM-20, pp. 912-922, Oct. 1972.

[24] R. G. Vaughan, "Signals in mobile communications: A review," IEEE Trans. Veh. Technol., vol. VT-35, pp. 133-145, Nov. 1986.

[25] T. Taga and K. Tsunekawa, "Performance analysis of a built-in planar inverted $F$ antenna for $800 \mathrm{MHz}$ band portable radio units," IEEE J. Selected Areas Commun., vol. SAC-35, pp. 921-929, June 1987.

[26] K. Kagoshima, K. Tsunekawa, and A. Ando, "Analysis of a plana inverted $F$ antenna fed by electromagnetic coupling," in 1992 IEEE AP-S Int. Symp. Dig., Chicago, July 18-25, 1992, pp. 1702-1705.

Michael A. Jensen (S'92) received the B.S. (summa cum laude) and M.S. degrees in electrical engineering from Brigham Young University (BYU), Provo, UT, in 1990 and 1991 , respectively. He is currently pursuing the Ph.D. degree in electrical engineering at the University of California, Los Angeles (UCLA).

From 1989 to 1991 he was a Graduate Research Assistant in the Lasers and Optics Laboratory, BYU. In 1990 he received a National Science Foundation Graduate Fellowship. Since 1991, he has been with

the Antenna Laboratory at UCLA as a Graduate Student Researcher. His main research interests include optical fiber communication, radiation and propagation for personal communications, numerical electromagnetics, and implementation of finite-difference schemes on massively parallel computer architectures.

Mr. Jensen is a member of Eta Kappa Nu and Tau Beta Pi. 
Yahya Rahmat-Samii (S'73-M'75-SM'79-F'85) received the M.S. and Ph.D. degrees in electrical engineering from the University of Illinois, Champaign-Urbana.

$\mathrm{He}$ is a Professor of Electrical Engineering at the University of California, Los Angeles. He has been a Senior Research Scientist at NASA's Jet Propulsion Laboratory/California Institute of Technology since 1978, where he contributed significantly to the advancement of antenna technology for space programs. He was a Guest Professor at the Technical

University of Denmark (TUD) in the summer of 1986 . He has also been a consultant to many aerospace companies.

Dr. Rahmat-Samii is a Fellow of IAE (1986) and was the 1984 recipient of the Henry Booker Award of URSI. He was appointed an IEEE Antennas and Propagation Society Distinguished Lecturer and presented lectures internationally. He was an elected IEEE AP-S AdCom member for the second term and has been an Associate Editor of the IEEE TRANSACTIONS on ANTEnNas and Propagation and the society's magazine. He is currently the elected Vice President of IEEE AP-S. He was the Chairman of the IEEE Antennas and Propagation Society of Los Angeles in 1987-1989. In 1989, his chapter received the Antennas and Propagation Best Chapter Award from the AP Society. He is one of the three International Editors of the IEE book series on Electromagnetics and Antennas. He is also one of the Editors of the Journal of Electromagnetic Waves and Applications. He is one of the Directors of the Antenna Measurements Technique Association (AMTA) and the Electromagnetics Society. He is listed in Who's Who in America, Who's Who in Frontiers of Science and Technology, and Who's Who in Engineering. He has authored or coauthored over 300 technical journal articles and conference papers and has written chapters in 12 books. He has made pioneering contributions to the developments of near-field planepolar and bipolar antenna measurements, microwave holographic diagnostics, mobile satellite communication antennas, reflector surface compensation, multireflector antenna diffraction analysis and synthesis, scattering and radiation from complex objects, RCS computations, singularity in dyadic Green's function, high-power microwave (HPM) antennas, EMP and aperture penetration, the Spectral Theory of Diffraction (STD), and GTD. For these contributions he has received numerous NASA Certificates of Recognitios and recently earned the JPL Team NASA's Distinguished Group Achievement Award. In 1992, he was the recipient of the Best Application Paper Award (Wheeler Award) for a paper published in 1991. In 1993, one of his Ph.D students was named the Most Outstanding Ph.D. Student at UCLA's School of Engineering and Applied Science and another received the Best Studen Paper Award at the 1993 IEEE AP-S Symposium. He is a member of Commissions A, B, and J of USNC/URSI, Sigma Xi, Eta Kappa Nu, and the Electromagnetics Academy. 